\title{
The Usefulness of a Preoperative Compact Imager, a Hand- Held $\gamma$-Camera for Breast Cancer Sentinel Node Biopsy: Final Results of a Prospective Double-Blind, Clinical Study
}

\author{
Khaldoun Kerrou ${ }^{1}$, Stéphanie Pitre ${ }^{2}$, Charles Coutant ${ }^{3}$, Roman Rouzier ${ }^{3}$, Pierre-Yves Ancel ${ }^{4,5}$, Cécile Lebeaux ${ }^{4,5}$, \\ Virginie Huchet ${ }^{1}$, Françoise Montravers ${ }^{1}$, Odile Pascal ${ }^{1}$, Marie-Alix-Duval ${ }^{2,6}$, Françoise Lefebvre ${ }^{2}$, Laurent Menard ${ }^{2}$, \\ Serge Uzan ${ }^{3}$, Yves Charon ${ }^{2}$, and Emmanuel Barranger ${ }^{3}$ \\ ${ }^{1}$ Nuclear Medicine Department, AP-HP Hôpital Tenon, Paris, France; ${ }^{2}$ Laboratoire IMNC-CNRS P7-P11, Paris, France; \\ ${ }^{3}$ Gynecology Department, AP-HP Hôpital Tenon, Paris, France; ${ }^{4}$ INSERM, UMR S953, Epidemiological Research Unit on Perinatal \\ Health and Women's and Children's Health, Hôpital Tenon, Paris, France; ${ }^{5}$ Université Pierre et Marie Curie Paris 06, UMR S 953, \\ Paris, France; and ${ }^{6}$ Université d'Evry Val d'Essonne, Evry, France
}

The aim of this study was to compare the effectiveness of a hand-held preoperative compact imager $(\mathrm{POCl})$ camera with conventional lymphoscintigraphy using a $\gamma$-camera for sentinel lymph node (SLN) detection in breast cancer. Methods: The main objective was to demonstrate the noninferiority of the $\mathrm{POCl}$ relative to conventional lymphoscintigraphy and to compare the number of SLNs detected by the 2 imaging devices. Our study, a clinical prospective, double-blind, noninferiority study, planned to include 200 patients with early breast cancer and started in January 2006. A standard SLN protocol (4 periareolar injections of $37 \mathrm{MBq}$ of $99 \mathrm{mTc}$-nanocolloids, $2 \mathrm{~h}$ before lymphoscintigraphy) was performed preoperatively using a conventional $\gamma$-camera and then the POCl camera. Scans were obtained by 2 different nuclear medicine physicians unaware of each other's results. The day after, in the operating room, the surgeon, after receiving the previous results, used the counting probe for surgical SLN biopsy. The number and localization of axillary SLNs obtained by lymphoscintigraphy and the $\mathrm{POCl}$ and the duration of the whole procedure were determined. Results: Among the 162 patients included, 138 were evaluable. The POCl detected more SLNs than did lymphoscintigraphy in 50 patients (36\%), the same number of in 54 patients (39\%), and fewer SLNs in 34 patients $(25 \%)$, representing $84(61 \%)$ discordant pairs. The noninferiority of preoperative compact imaging of axillary SLNs numbers was found to be statistically significant $(95 \%$ confidence interval, $30 \%-52 \%, P=0.025)$ using the McNemar test. The duration of acquisition was shorter using the $\mathrm{POCl}(<10 \mathrm{~min}$ in $84 \%$ [ $n=117$ ] of patients; mean, $7.5 \pm 3.3 \mathrm{~min}$ ) than lymphoscintigraphy (13\% [ $n=18]$ of patients; mean, $15.7 \pm 3.4 \mathrm{~min})$, with $P<0.001$ using the McNemar test for paired proportions. Conclusion: Preoperative compact imaging using a hand-held camera was able to predict the number and localization of breast cancer SLNs and was not inferior to conventional lymphoscintigraphy in this study. Further studies will determine whether preoperative compact imaging could replace lymphoscintigraphy,

\footnotetext{
Received Mar. 14, 2011; revision accepted Jun. 20, 2011.

For correspondance or reprints contact: Khaldoun Kerrou, Assistance Publique-Hôpitaux de Paris, Hôpital Tenon, 4, Rue de la Chine, 75020 Paris, France.

E-mail: khaldoun.kerrou@tnn.aphp.fr

Published online Aug. 17, 2011.

COPYRIGHT @ 2011 by the Society of Nuclear Medicine, Inc.
}

especially in surgical centers without an on-site nuclear medicine department.

Key Words: breast; instrumentation; lymphoscintigraphy; handheld gamma camera; sentinel node

J Nucl Med 2011; 52:1346-1353

DOI: 10.2967/jnumed.111.090464

$\mathbf{T}$ he disease status of the axillary lymph nodes (LNs) is the most significant prognostic factor for patients with early-stage breast cancer (BC) (1). Thus, reliance on histologic examination of the LNs remains the most accurate method for assessing spread of disease-important not only for staging and prognosis but also for treatment selection guiding (1). For many years, the use of sentinel lymph node (SLN) identification and a sampling procedure referred to as sentinel node biopsy (SNB) has progressively reduced the need for axillary lymph node dissection (2) and avoided its associated morbidity. This procedure has now become practiced worldwide (3).

Currently, dual detection of SLN with blue dye and radioactive colloids is considered the reference method $(4,5)$. Dual detection not only improves the detection rate (estimated at $\sim 95 \%$ ) but also reduces the risk of false-negative results and of axillary relapse in the cases in which axillary dissection is not undertaken because the SLN is nonmetastatic. False-negative results are the main weakness of this technique, because they can potentially lead to undertreatment. When radiolabeling is used, international experts (5) recommend not only dual detection but also preoperative lymphoscintigraphy to predict the success of the procedure, establish a precise map of the hot SLN (axillary or extraaxillary), and determine the number of SLNs detected. Preoperative lymphoscintigraphy also aids the surgeon by showing the precise axillary location of the SLN (base of the axilla or higher). A recent series (6) of 1,201 patients showed that the visualization of axillary SLN procedure by preoperative lymphoscintigraphy was asso- 
ciated with a higher detection rate $(98.7 \%$ with vs. $93 \%$ without; $P<0.001$ ). However, no randomized studies have so far compared the detection and false-negative rates with and without the use of preoperative lymphoscintigraphy.

Thus, many teams that are skeptical about the value of preoperative lymphoscintigraphy (7) believe this technique complicates the SLN procedure without providing any real benefit. This attitude is partly due to the scarcity in several countries of medical centers possessing nuclear medicine departments and to cost issues. The $\gamma$-cameras used for preoperative lymphoscintigraphy in the nuclear medicine departments are often in use for other medical examinations. Moreover, the examination takes about 15-35 min, during which the patient has to remain immobile. For teams without easy access to preoperative lymphoscintigraphy, options include limiting the procedure to patients most likely to benefit from it, using blue dye alone (not the optimal technique), or undertaking the procedure without preoperative scintigraphy, which is contrary to international recommendations.

In this context, many laboratories and companies have developed hand-held $\gamma$-cameras with different technologies (8-15). Some of them have been evaluated in different clinical applications such as parathyroid imaging (16-19), brain $(20)$, bone tumors $(21,22)$, and SNB procedures in BC $(21,23-30)$ and more recently in melanoma and gynecologic cancers (31), head and neck cancers (32), and prostate (33) or other urologic cancers (34). Few prospective and masked clinical studies, however, have been conducted to ensure that these devices performed as well as conventional $\gamma$-cameras in clinical routine practice.

The aim of this present clinical trial was to evaluate the ability of hand-held $\gamma$-cameras to simplify preoperative lymphoscintigraphy and optimize patient throughput, particularly in hospitals that lack a nuclear medicine department. This clinical trial was performed with a hand-held $\gamma$-camera prototype called preoperative compact imager (POCI) developed by the Nuclear Physics Institute and the Imaging and Modeling in Neurobiology and Cancerology Laboratory (24).

We designed a prospective study to compare the performance of the POCI device with conventional preoperative lymphoscintigraphy using a $\gamma$-camera in patients with early $\mathrm{BC}$ requiring SNB after combined detection. The objective was to assess if preoperative compact imaging could replace classic preoperative lymphoscintigraphy, without affecting patient outcome. The POCI, if shown to be noninferior to conventional preoperative lymphoscintigraphy regarding the number of SLNs detected, could be used routinely to create a radioactive map of the axillary or extraaxillary areas. Moreover, the use of the POCI to perform lymphoscintigraphy could improve access to dual-detection SLN procedures.

\section{MATERIALS AND METHODS}

\section{Materials}

The POCI device was designed to be easy to handle and ensure accurate and real-time radioguided detection of tumors during surgery (Fig. 1). The simplicity of its clinical use is due to a pedal footswitch that can be pressed to start and stop the image acquisition. The POCI device combines compactness, lightness, and high performance with a 40-mm-diameter field of view. The imaging head comprises a high-resolution parallel-hole lead collimator coupled to a 3-mm-thick continuous $\mathrm{CsI}(\mathrm{Na})$ crystal plate. The power and electronic module is connected to the camera by a 5-m-long wire cable. Data and image processing are performed in real time by a personal computer.

The camera has an outer diameter of $9.5 \mathrm{~cm}$, a thickness of 9 $\mathrm{cm}$, and a weight of $1.2 \mathrm{~kg}$.

The physical performance of the POCI device is well detailed in the article published by Pitre et al. (24). The spatial resolution is $2.3 \mathrm{~mm}$ in full width at half maximum at $140 \mathrm{keV}$, much smaller than that of $\gamma$-cameras used in nuclear medicine departments $(\sim 1$ $\mathrm{cm}$ for SLN localization). The full-width-at-half-maximum energy resolution of the $140-\mathrm{keV}$ full-energy peak is $28 \%$. The sensitivity is $290 \mathrm{cps} / \mathrm{MBq}$. An image of the SLN can be acquired in a few seconds using a total typical injected dose for lymphatic mapping of about $150 \mathrm{MBq}$, and $1 \%$ diffuses into 1 SLN. Associated to a surface of analysis of $13 \mathrm{~cm}^{2}$, this good sensitivity allows investigation of the axilla or operative wound, without stretching the duration of surgical tumor ablation (24).

\section{Patients}

The patients were older than $18 \mathrm{y}$, had early BC confirmed by histopathology on core biopsy or cytopathology, a unifocal tumor no more than $20 \mathrm{~mm}$ in largest dimension as measured by mammography or ultrasound, and no clinically palpable axillary LN. All patients gave informed written consent to the SLN biopsy procedure and axillary LN detection using the POCI.

Exclusion criteria patients were an age younger than $18 \mathrm{y}$, multifocal BC, prior neoadjuvant chemotherapy, clinically palpable axillary LN, pregnancy, and a prior history of surgery of the axilla or breast plastic surgery.
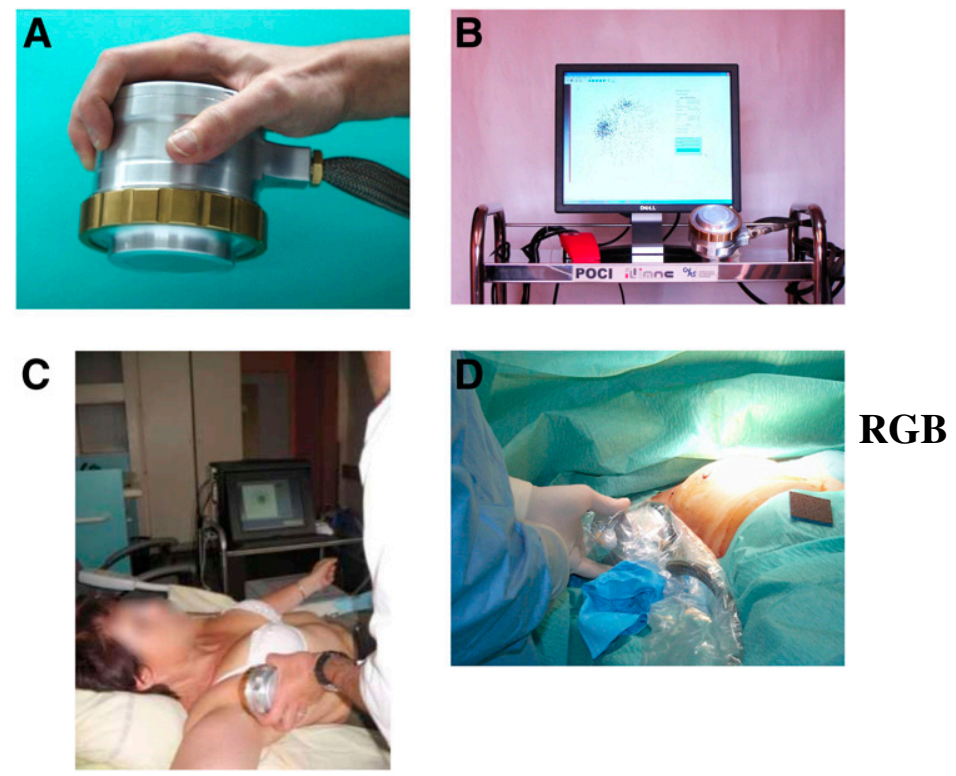

FIGURE 1. (A) Handheld POCl camera. (B) POCl device on its clinical trolley. (C) $\mathrm{POCl}$ device in patient room. (D) $\mathrm{POCl}$ device in operating room. 


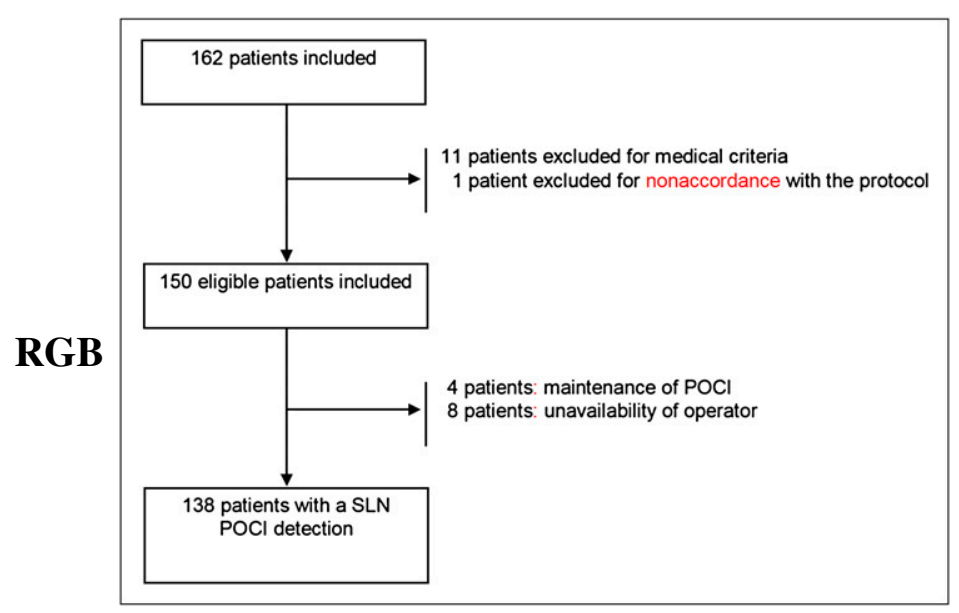

FIGURE 2. Flowchart of patients.

\section{Sample Size}

Because each patient was her own control in this study, a concordant pair was defined as identical numbers of radioactive SLNs detected by lymphoscintigraphy and preoperative compact imaging for the same patient. A discordant pair was defined as different numbers of radioactive SLNs detected by lymphoscintigraphy and preoperative compact imaging.

The number of patients to include was calculated according to the following considerations and hypotheses. It was postulated that in no more than $3 \%$ of patients (a clinically acceptable percentage) should preoperative compact imaging be shown inferior to lymphoscintigraphy. The expected number of discordant pairs was estimated to be around $10 \%$ and the noninferiority margin to be $30 \%$, meaning that the difference of performance showing an inferiority of preoperative compact imaging relative to lymphoscintigraphy would have concerned only $30 \% \times 10 \%=3 \%$ of patients. However, because no previous publication or preliminary series had estimated the percentage of discordant pairs, it was decided to adjust the noninferiority margin accordingly with the obtained results, so as to not exceed the $3 \%$ determined as clinically acceptable.

According to these hypotheses, the minimal number of patients to be included was 171 with the hypothesis of $10 \%$ discordant pairs and $30 \%$ margin. The minimal number was calculated by the McNemar test applied to the noninferiority formula: $n=(1,645+$ $0.84)^{2} / 0.3^{2} \times\left(0.5^{2} / 10 \%\right)(35)$.

\section{Main Judgment Criteria}

The performances of the POCI and lymphoscintigraphy were evaluated through direct comparison of the numbers of SLN obtained from both modalities on the same patient and the discordance of these results. For each patient, conventional lymphoscintigraphy and preoperative compact imaging detection were performed on the same preoperative day by 2 different nuclear medicine physicians, unaware of each other's results. The patients presenting discordance of the numbers of SLNs detected between the 2 methods were analyzed, and those showing fewer SLNs with preoperative compact imaging detection than with conventional lymphoscintigraphy were further computed.

\section{SLN Protocol}

The SLN protocol consists of four 0.2-mL periareolar injections, each containing $37 \mathrm{MBq}$ of ${ }^{99 \mathrm{~m}} \mathrm{Tc}$-labeled nanocolloids (Nanocis; CIS Bio International), administered $2 \mathrm{~h}$ before lymphoscintigraphy in the nuclear medicine department. Lymphoscintigraphy was performed by a standard triple-head $\gamma$-camera (IRIX Marconi; Philips) equipped with high-resolution low-energy collimators. Anterior and posterior simultaneous views, with a $256 \times 256$ matrix and $20 \%$ energy window centered on the $140-\mathrm{keV}{ }^{99 \mathrm{~m}} \mathrm{Tc}$ photopeak, were acquired in $5 \mathrm{~min}$. In the case of radioactive SLN visualization, a transmission image with uniform ${ }^{57} \mathrm{Co}$ source in the projection best showing SLNs was acquired in 5 min to provide anatomic landmarks for the surgeon. A second attempt was made 30 min later if no SLN was detected after the first acquisition.

Then, preoperative compact imaging was performed in the patient's room in the gynecology department between $5 \mathrm{~min}$ and $2 \mathrm{~h}$ after lymphoscintigraphy. The whole axillary area was scanned with the POCI in contact with the patient's skin, using at least 5- to 10 -s acquisitions with a $256 \times 256$ matrix. The POCI device was moved to scan the whole axillary area, and a screening for extraaxillary SLNs was then performed, focusing on the homolateral internal mammary and the infra- and the supraclavicular areas.

\section{Statistical Analysis}

As recommended for paired samples, only patients with discordant SLN numbers between the 2 modalities were analyzed. The repartition of the different pairs was defined as $n 1$ (the number of patients in whom the POCI identified fewer SLNs than did lymphoscintigraphy), n2 (the number of patients in whom the POCI and lymphoscintigraphy identified the same number of nodes), and $\mathrm{n} 3$ (the number of patients in whom the POCI was more successful than lymphoscintigraphy). The statistical McNemar test was adapted to noninferiority trials (36), taking into account the noninferiority margin in the calculation of the theoretic sample size required for the test. A judgment of noninferiority was made if the distribution between $\mathrm{n} 1$ and $\mathrm{n} 3$ was close to $50 \% / 50 \%$, which was determined according to the margin of noninferiority $\Delta$.

$$
\begin{array}{r}
\mathrm{H}_{0}=\text { POCI }<\text { lymphoscintigraphy } \\
\mathrm{H}_{1}=\text { POCI }>\text { lymphoscintigraphy } \\
\qquad z=\frac{n_{1}-n_{3}-n \Delta}{\sqrt{n_{3}+n_{1}-n \Delta^{2}}} .
\end{array}
$$

\section{RESULTS}

Between January 2006 and February 2008, 162 consecutive patients were enrolled and registered in the database. The flowchart of patients is shown in Figure 2. Eleven patients were excluded a posteriori on the basis of the exclusion criteria: size of tumor superior to $20 \mathrm{~mm} \mathrm{(5}$ patients), clinically palpable axillary LN (2 patients), prior history of breast plastic surgery (2 patients), multifocal BC (1 patient), and benign tumor (1 patient). The protocol was not followed for 1 patient (1 exclusion).

Eight patients were ineligible because of the unavailability of the operator and 4 more because the POCI device was undergoing maintenance.

Because of the 12 exclusions and the 12 other noneligible patients, the results regarding the main judgment criteria are for 138 patients. 
TABLE 1

Patient and Tumor Characteristics

\begin{tabular}{lcc}
\hline \multicolumn{1}{c}{ Characteristic... } & Patients with available data $(n)$ & Result \\
\hline For patients & 138 & $58 \pm 12$ \\
Age at diagnosis $(\mathrm{y})$ & 130 & $25 \pm 5$ \\
Body mass index $\left(\mathrm{kg} / \mathrm{m}^{2}\right)$ & 137 & $13(9.5)$ \\
$\quad$ Prior history of cancer $(n)$ & 138 & $47(34)$ \\
$\quad$ Familial history of BC $(n)$ & 138 & $14.3(8.7)$ \\
For tumors & 138 & $83(60)$ \\
Final size of tumor on histology $(\mathrm{mm})$ & & $19(14)$ \\
Histology subtypes $(n)$ & & $25(18)$ \\
$\quad$ Invasive ductal carcinoma & & $11(8)$ \\
Invasive lobular carcinoma & & \\
Intra ductal carcinoma & 116 & $54(47)$ \\
Others & & $50(43)$ \\
Scarff-Bloom-Richardson grade $(n)$ & & $12(10)$ \\
I & & $15(13)$ \\
II & 115 & $83(71)$ \\
Hill & 117 & $105(89)$ \\
Positive progesterone receptors $(>10 \%)(n)$ & 118 & $10(8.5)$ \\
Positive estrogens receptors $(>10 \%)(n)$ & 117 & $126(91)$ \\
Cerb2 overexpression $(n)$ & & $12(9)$
\end{tabular}

Data in parentheses are percentages.

The clinical characteristics of the patients and tumors are given in Table 1. Patients' mean age was $58 \mathrm{y}(\mathrm{SD}=12)$, with a mean body mass index of $25(\mathrm{SD}=5)$. All tumors were unilateral and of $\mathrm{T} 1$ type. The mean size of the tumors measured in histology was $14.3 \mathrm{~mm}(\mathrm{SD}=8.7)$ in their greatest dimension. The histologic subtype was invasive ductal carcinoma for 83 patients (60\%), intraductal carcinoma in situ for 25 patients (18\%), invasive lobular carcinoma for 19 patients (14\%), and other histologic BC subtype for 11 patients (8\%). Twelve patients (9\%) had immediate mastectomy.

\section{Lymphoscintigraphy Versus Preoperative Compact Imaging}

Results of lymphoscintigraphy, compared with preoperative compact imaging, are detailed in Tables 2-5.

Two hundred thirty-six axillary SLNs were identified by lymphoscintigraphy (mean, 1.7; range, 0-6 per patient). Twenty-nine (range, 1-3) extraaxillary SLNs were identi- fied in 16 patients. After lymphoscintigraphy, the day before surgery, the POCI identified 256 axillary SLNs (mean, 1.9; range, 0-6 per patient). Fifteen (1-3) extraaxillary SLNs were identified in 10 patients. Considering that 291 (mean, 2.3; range, 0-6 per patient) radioactive axillary SLNs were removed from the 138 patients as reference, the preoperative sensitivity for axillary SLN detection was $81 \%$ (95\% confidence interval [CI], 76.5\%-85.5\%) for lymphoscintigraphy and $88 \%(95 \% \mathrm{CI}, 84 \%-92 \%)$ for preoperative compact imaging. No SLN was seen in 6 patients for lymphoscintigraphy and 7 patients for the POCI ( 3 cases in common); thus, the success rate was $96 \%$ (132/138) for lymphoscintigraphy and 95\% (131/138) for the POCI.

For axillary SLN detection, the results for lymphoscintigraphy and the POCI matched in 39\% of the patients (54/ 138 ) and were discordant for the remaining $61 \%$ of patients (84/138). Because of our postulation preoperative compact imaging should be inferior to lymphoscintigraphy in no

TABLE 2

SLN Identification

\begin{tabular}{|c|c|c|c|c|c|c|c|c|c|}
\hline \multirow[b]{2}{*}{ Node } & \multicolumn{3}{|c|}{ Lymphoscintigraphy } & \multicolumn{3}{|c|}{$\mathrm{POCl}$} & \multicolumn{3}{|c|}{ Surgery } \\
\hline & $n$ & Mean & Range & $n$ & Mean & Range & $n$ & Mean & Range \\
\hline Axillary & 236 & 1.7 & $0-6$ & 256 & 1.9 & $0-6$ & 291 & 2.3 & $0-6$ \\
\hline Extraaxillary & 29 & 16 patients & $1-3$ & 15 & 10 patients & $1-3$ & - & - & - \\
\hline SN not identified & 6 & & & 7 & & & - & - & - \\
\hline
\end{tabular}


TABLE 3

Number of Patients with Axillary and Extraaxillary Nodes Identified by Classic Lymphoscintigraphy and POCI on Day Before Surgery

\begin{tabular}{|c|c|c|c|c|}
\hline Node & $\begin{array}{l}\mathrm{POCl} \text { identified fewer LNs than did } \\
\text { lymphoscintigraphy }\end{array}$ & $\begin{array}{l}\mathrm{POCI} \text { performed equally as well as } \\
\text { lymphoscintigraphy }\end{array}$ & $\begin{array}{l}\text { POCI identified more LNs than did } \\
\text { lymphoscintigraphy }\end{array}$ & $\begin{array}{c}\text { Total } \\
(n)\end{array}$ \\
\hline Axillary & $34(25)$ & $54(39)$ & $50(36)$ & 138 \\
\hline Extraaxillary & $14(10)$ & $120(87)$ & $4(3)$ & 138 \\
\hline
\end{tabular}

more than $3 \%$ of patients, the noninferiority margin was therefore adjusted to $\Delta=5 \%$, to respect the limit of $61 \% \times$ $5 \%=3 \%$ of patients limit.

In 50 patients $(36 \%)$, the POCI identified more SLNs than did lymphoscintigraphy, representing 60\% $(95 \% \mathrm{CI}$, $49 \%-70 \%)$ of the discordant pairs. In 34 patients $(25 \%)$, lymphoscintigraphy identified more SLNs than did the POCI, representing $40 \%$ (95\% CI, 30\%-52\%) of the discordant pairs. Because the upper limit of the $95 \%$ CI $(52 \%)$ was less than $55 \%(50 \%+\Delta)$ with a $P$ value less than 0.05 , the noninferiority of preoperative compact imaging, compared with lymphoscintigraphy, was proven.

The durations of lymphoscintigraphy and preoperative compact imaging examinations were also compared. Lymphoscintigraphy lasted a mean of $15.7 \mathrm{~min}(\mathrm{SD}=3.3)$, whereas with the POCI the nodes screening lasted a mean of only $7.5 \mathrm{~min}(\mathrm{SD}=1.7)$ including the extraaxillary area screening. The SLN detection with lymphoscintigraphy lasted the minimal acquisition time of $10 \mathrm{~min}$ or less in 18 patients $(13 \%)$, and the whole detection with the POCI, including extraaxillary SLNs, lasted less than 10 min in 117 patients $(84 \%)$. The difference was statistically significant, with a $P$ value of 0.001 (McNemar test for paired series).

\section{DISCUSSION}

Our study shows that the characteristics and performance of the POCI are adequate for BC SLNs detection. For $15 \mathrm{y}$, many research laboratories have built miniaturized imagers $(8,10-13,15)$, with different designs and performances. Today,

TABLE 4

Results for Discordant Pairs of Patients

\begin{tabular}{ccc}
\hline \multicolumn{1}{c}{ Result } & $\begin{array}{c}\text { Axillary } \\
\text { nodes }\end{array}$ & $\begin{array}{c}\text { Extraaxillary } \\
\text { nodes }\end{array}$ \\
\hline No. of discordant pairs & $84(61)$ & $18(13)$ \\
Noninferiority margin $\Delta$ & $5 \%$ & $23 \%$ \\
$\mathrm{POCl}<$ & 34 & 14 \\
lymphoscintigraphy & & \\
$\%$ & $40 \%$ & $78 \%$ \\
$95 \% \mathrm{Cl}$ & $30 \%-52 \%$ & $52 \%-94 \%$ \\
\hline
\end{tabular}

*No. of patients in whom POCl has identified fewer LNs than lymphoscintigraphy.

$P=0.025$ (McNemar test) with $\Delta=0.05$ for axillary vs. extraaxillary. Data in parentheses are percentages. the performances of these imagers have converged to optimize the detection efficiency, in order to reduce the duration of acquisition. To achieve that feature, some cameras are even supplied with pinhole collimators, which can create difficulty with interpreting images because of zoom effects inherent to focus depth (37). In our case, the POCI device is equipped with a high-sensitivity parallel-hole collimator that allows image acquisition in 5-10 s. Once familiar with the POCI camera, the operator can identify and scan the region of interest more rapidly because nonradioactive areas can be skipped.

From a design and conception point of view, 2 different types of imagers coexist: those with small fields of view (5-cm maximum in their larger dimension) and those with $10 \mathrm{~cm}$ or more on their longest side. The devices of the first group have the advantage of being compact and are therefore portable (with no arm), meaning that they can easily be used in nuclear medicine departments, operating rooms, and also the patient's room. Their small analysis areas require performing rigorous scanning of the region of interest. Imagers belonging to the second group allow a picture of the whole axilla to be taken in 1 shot. Nevertheless, the large dimensions of these imagers prevent their head from being in close contact with the axilla and the consequent better resolution and precise topographic localization, and their weight is often greater than $2 \mathrm{~kg}$, complicating their use and handling and imposing the necessity of an articulated arm.

TABLE 5

Duration of Examinations

\begin{tabular}{ccc}
\hline & $\begin{array}{c}\text { Lymphoscintigraphy } \\
(n=129)\end{array}$ & $\begin{array}{c}\mathrm{POCl} \\
(n=138)\end{array}$
\end{tabular}

*Durations were discrete data: $10,15,20,25$, or 30 min.

${ }^{\star \star}$ Comparison McNemar test of percentage for paired series. Data in parentheses are percentages. 
Since 1997, our team has preferred portability and chosen to develop POCI (38) to offer the better ergonomics to the operator.

The clinical trials reported to date in the literature about compact $\gamma$-cameras are scarce $(10,25,28,30,39,40)$, and our trial (which started in January 2006) is one of the first and biggest studies so far. Until now, the similar studies have investigated the use of compact $\gamma$-cameras versus surgical counting probes and have provided only a proof of concept or a descriptive analysis of the preoperative detection. All of these studies have not supported their results with comparative prospective studies and statistical tests, except the Italian team (30), which used a Student $t$ test in a prospective study but in unpaired groups of patients, comparing the surgical probe in one group and the surgical probe plus the imaging probe in the other group, in the operating room. All of these studies concluded that compact $\gamma$-cameras had the potential to perform lymphoscintigraphy and to replace the standard $\gamma$-cameras.

Our trial was the first, to our knowledge, comparing the performances of conventional lymphoscintigraphy using a $\gamma$-camera and preoperative compact imaging using a handhand $\gamma$-camera on a large number of patients $(n>100)$. A similar comparative study was reported in 2005 by Goto et al. (40), but it was performed on only 19 patients. Furthermore, our trial was the first to be conducted in a prospective and masked manner, comparing lymphoscintigraphy and preoperative compact imaging in the same 138 patients. Our aim was to give a quantitative answer about the potential of a compact imager to replace conventional $\gamma$-cameras. Therefore, we chose to conduct a noninferiority trial between lymphoscintigraphy and the POCI for localization and counting SLNs. Noninferiority trials as frequency tests for treatment validation are increasing in the literature. Our study shows that the 2 methods are not very different and that the difference in performance remains smaller than the usual clinically acceptable percentage of $5 \%$. We have chosen to reduce this percentage to $3 \%$ to more stringently evaluate this new-generation portable detector, with the ulterior motive of its further use in clinical practice.

The noninferiority of the POCI camera, compared with the standard $\gamma$-camera, was clearly demonstrated in our study $(P=0.025$; McNemar adapted paired test for noninferiority) and was achieved in the designed setting or preoperative screening on the day before surgery. One surprise in our study was that the number of discordant pairs reached $61 \%$. Before the study, it was estimated to be around $10 \%$ and the noninferiority margin to be $30 \%$ to respect the condition that in no more than $3 \%$ of patients $(10 \% \times 30 \%)$ should preoperative compact imaging be shown inferior to lymphoscintigraphy. As no prior publications or preliminary series had estimated the percentage of discordant pairs, we decided to adjust the noninferiority margin according to the obtained results in order to not exceed the $3 \%$ limit, possibly leading to a different and probably bigger sample size than previously known and thus potentially reducing the power of our study. But despite this theoretic reduction in power and larger than expected number of discordant pairs, the non- inferiority of the POCI, compared with lymphoscintigraphy, was achieved, with an inferiority limit of 3\%-a percentage less than the usually clinically acceptable $5 \%$ margin.

Nuclear medicine physicians who used the POCI identified more SLNs than they did with lymphoscintigraphy in 50 of 138 patients $(36 \%)$, and the opposite was seen in only 34 patients (25\%). For example, as Figure 3 shows, 5 SLNs close to each other are clearly detected on the image from the POCI device, whereas classic lymphoscintigraphy showed only 1 SLN. The excellent spatial resolution of the POCI camera allowed a more precise counting of SLNs.

For extraaxillary SLN detection, the clinical results of the POCI were inferior to those of lymphoscintigraphy, and noninferiority was not found but was not a study endpoint. First, the POCI device has a small field of view $\left(13 \mathrm{~cm}^{2}\right)$, which does not allow a 1-shot screening of extraaxillary areas. Lymphoscintigraphy, however, with the large-fieldof-view $\gamma$-camera, allowed the detection of extraaxillary SLNs in the same acquisition as for axillary ones. Second, the time that nuclear medicine physicians spent screening the extraaxillary areas could have been shorter than for the axilla because the extraaxillary SLNs are not excised either for biopsy in our routine practice or for this study.

The additional advantage to performing lymphoscintigraphy with this device was that the examination was much shorter, even if a meticulous scan of the axilla and of the extraaxillary areas were to be performed. This shorter duration could be reasonably reduced even further by avoiding the screening for extraaxillary areas because the eventual detection of a SLN in these locations has no clinical impact in routine practice.

The general recommendations for BC require lymphoscintigraphy in the SLN biopsy procedure $(4,5)$. This examination has some limitations because of the use of standard $\gamma$-cameras - the main limitation being the need for a nuclear medicine department - and because of the duration of the examination, which sometimes keeps the patient a couple of hours in the nuclear medicine department. In addition, the benefit of portable $\gamma$-cameras has been a matter of debate (7). The hope is that these new portable $\gamma$-cameras will replace the standard $\gamma$-cameras, consequently making the

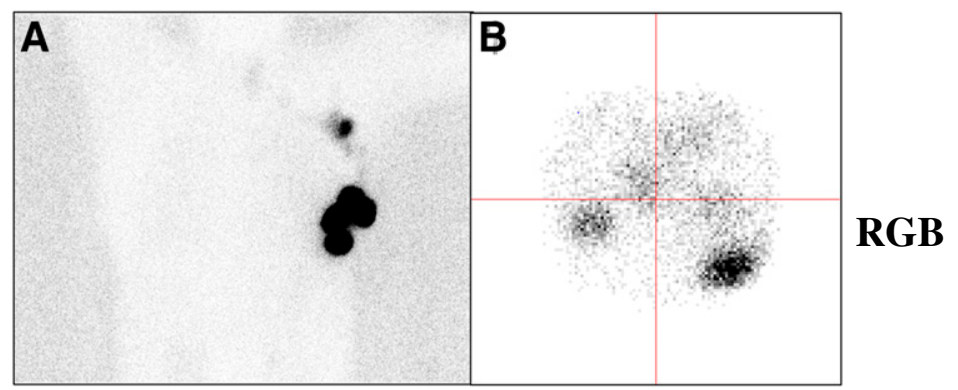

FIGURE 3. Lymphoscintigraphy in patient 73. (A) Lymphoscintigraphy performed with standard $\gamma$-camera, which identified 1 SLN. Image acquisition time was 5 min. (B) Lymphoscintigraphy performed by $\mathrm{POCl}$ device a few minutes later. Five SLNs were clearly identified. Image acquisition time was $79 \mathrm{~s}$. 
procedure easier and facilitating access to SLN biopsies for patients in hospitals lacking on-site nuclear medicine departments.

This study had some limitations. The higher than expected number of discordant pairs might have been a limitation and was already discussed. In addition, the fact that more than $90 \%$ of the POCI SLN detections were performed by the same operator could constitute a positive bias, because a learning curve, even if short, could exist for using this device. This situation might be different in real-life and with multioperator use, but like all the portable cameras, our POCI device is userfriendly and intuitively handled and controlled after 1 procedure.

Because preoperative compact imaging was always performed after conventional lymphoscintigraphy, more uptake might have migrated to additional SLNs at the time of preoperative compact imaging. The current study design may thus be biased into a higher number of SLNs detected by the POCI than with lymphoscintigraphy, therefore reducing the calculated inferiority of the POCI. To remove this bias, the order of imaging could have also been randomized.

Finally, these results were obtained in a single center; despite the prospective and masked design, this can be another limitation to the evaluation of the POCI performance. A multicenter prospective and masked phase III-like trial could definitively answer this question.

\section{CONCLUSION}

We have shown in a large-scale, prospective, and masked study that the POCI was able to predict the number and localization of BC axillary SLNs and was not inferior to conventional lymphoscintigraphy in a statistically significant manner.

These performances were also achievable for shorter acquisitions than are used for conventional lymphoscintigraphy. The POCI device is therefore a reliable tool to replace standard $\gamma$-cameras in clinical practice, especially in surgical centers without on-site nuclear medicine departments.

\section{DISCLOSURE STATEMENT}

The costs of publication of this article were defrayed in part by the payment of page charges. Therefore, and solely to indicate this fact, this article is hereby marked "advertisement" in accordance with 18 USC section 1734.

\section{ACKNOWLEDGMENTS}

We gratefully acknowledge helpful discussion with Dr. Joseph Gligorov and thank Professor Jean-Noël Talbot for continuous support. This study was supported by a grant from the Programme Hospitalier de Recherche Clinique of the French Ministry of Health (PHRC no. P040417). No potential conflict of interest relevant to this article was reported.

\section{REFERENCES}

1. Lyman GH, Giuliano AE, Somerfield MR, et al. American Society of Clinical Oncology guideline recommendations for sentinel lymph node biopsy in earlystage breast cancer. J Clin Oncol. 2005;23:7703-7720.

2. Giuliano AE. Intradermal blue dye to identify sentinel lymph node in breast cancer. Lancet. 1997;350:958.

3. Edge SB, Niland JC, Bookman MA, et al. Emergence of sentinel node biopsy in breast cancer as standard-of-care in academic comprehensive cancer centers. J Natl Cancer Inst. 2003;95:1514-1521.

4. Miltenburg DM, Millar C, Karamlou TB, Brunicardi FC. Meta-analysis of sentinel lymphnode biopsy in breast cancer. J Surg Res. 1999;84:138-142.

5. Schwartz GF, Giuliano AE, Veronesi U. Proceedings of the consensus conference on the role of sentinel lymph node biopsy in carcinoma of the breast, April 1922, 2001, Philadelphia, Pennsylvania. Cancer. 2002;94:2542-2551.

6. Kawase K, Gayed IW, Hunt KK, et al. Use of lymphoscintigraphy defines lymphatic drainage patterns before sentinel lymph node biopsy for breast cancer. J Am Coll Surg. 2006;203:64-72.

7. Burak WE Jr, Walker MJ, Yee LD, et al. Routine preoperative lymphoscintigraphy is not necessary prior to sentinel node biopsy for breast cancer. Am J Surg. 1999; 177:445-449.

8. Abe A, Takahashi N, Lee J, et al. Performance evaluation of a hand-held, semiconductor (CdZnTe)-based gamma-camera. Eur J Nucl Med Mol Imaging. 2003; 30:805-811.

9. Hoffman EJ, Tornai MP, Levin CS, MacDonald LR, Siegel S. Gamma and beta intra-operative imaging probes. Nucl Instrum Methods Phys Res A. 1997;392: 324-329.

10. Olcott P, Pratx G, Johnson D, Mansouri M, Mittra E, Levin C. Clinical feasibility of a high sensitivity intra-operative hand-held gamma-camera (IHGC) for sentinel lymph node biopsy (SLNB). J Nucl Med. 2009;50(suppl 2):510P.

11. Pani R, Pellegrini R, Cinti MN, et al. New devices for imaging in nuclear medicine. Cancer Biother Radiopharm. 2004;19:121-128.

12. Porras E, Escat B, Benlloch JM, et al. Portable mini gamma-camera for medical applications. Nucl Instrum Methods Phys Res A. 2002;486:186-190.

13. Salvador S, Bekaert V, Mathelin C, Guyonnet JL, Huss D. An operative gammacamera for sentinel lymph node procedure in case of breast cancer. $J$ Instrum. 2007;2:07003.

14. Tsuchimochi M, Hayama K, Oda T, Togashi M, Sakahara H. Evaluation of the efficacy of a small CdTe gamma-camera for sentinel lymph node biopsy. J Nucl Med. 2008;49:956-962.

15. Tsuchimochi M, Sakahara H, Hayama K, et al. A prototype small CdTe gammacamera for radioguided surgery and other imaging applications. Eur J Nucl Med Mol Imaging. 2003;30:1605-1614.

16. Cassinello $\mathrm{N}$, Ortega J, Lledo $\mathrm{S}$. Intraoperative real-time ${ }^{99 \mathrm{~m}} \mathrm{Tc}$-sestamibi scintigraphy with miniature gamma-camera allows minimally invasive parathyroidectomy without ioPTH determination in primary hyperparathyroidism. Langenbecks Arch Surg. 2009;394:869-874.

17. Lumachi F, Iacobone M, Favia G. Minimally invasive radioguided parathyroidectomy. Ann Ital Chir. 2003;74:413-416.

18. Ortega J, Ferrer-Rebolleda J, Cassinello N, Lledo S. Potential role of a new handheld miniature gamma-camera in performing minimally invasive parathyroidectomy. Eur J Nucl Med Mol Imaging. 2007;34:165-169.

19. Rubello D, Mariani G. Hand-held gamma probe or hand-held miniature gammacamera for minimally invasive parathyroidectomy: competition, evolution or synergy? Eur J Nucl Med Mol Imaging. 2007;34:162-164.

20. Calvo Moron C, de la Riva Perez PA, Cambil Molina T, Alvarez Marquez E, Castro Montano J. Brain perfusion image with a portable mini-gamma camera (Sentinella) in brain death [in Spanish]. Rev Esp Med Nucl. 2009;28:83-84.

21. Schillaci O, D'Errico G, Scafe R, et al. Sentinel node detection with imaging probe. Tumori. 2002;88:S32-S35.

22. Osebold WR, Lester EL, Hurley JH, Vincent RL. Intraoperative use of the mobile gamma-camera in localizing and excising osteoid osteomas of the spine. Spine (Phila Pa 1976). 1993;18:1816-1828.

23. Scafe R, Soluri A, Amanti C, et al. Clinical use of an imaging probe in breast cancer surgery. Tumori. 2002;88:S35-S37.

24. Pitre S, Menard L, Ricard M, Solal M, Garbay JR, Charon Y. A hand-held imaging probe for radio-guided surgery: physical performance and preliminary clinical experience. Eur J Nucl Med Mol Imaging. 2003;30:339-343.

25. Motomura K, Noguchi A, Hashizume T, et al. Usefulness of a solid-state gammacamera for sentinel node identification in patients with breast cancer. J Surg Oncol. 2005;89:12-17.

26. Barranger E, Kerrou K, Pitre S, Duval MA, Charon Y, Uzan S. Place of a handheld gamma-camera (POCI) in the breast cancer sentinel node biopsy. Breast. 2007; 16:443-444. 
27. Mathelin C, Salvador S, Croce S, Andriamisandratsoa N, Huss D, Guyonnet JL. Optimization of sentinel lymph node biopsy in breast cancer using an operative gamma-camera. World J Surg Oncol. 2007;5:132.

28. Mathelin C, Salvador S, Bekaert V, et al. A new intraoperative gamma-camera for the sentinel lymph node procedure in breast cancer. Anticancer Res. 2008;28 (5B):2859-2864.

29. Paredes P, Vidal-Sicart S, Zanon G, et al. Radioguided occult lesion localisation in breast cancer using an intraoperative portable gamma-camera: first results. Eur J Nucl Med Mol Imaging. 2008;35:230-235.

30. Scopinaro F, Tofani A, di Santo G, et al. High-resolution, hand-held camera for sentinel-node detection. Cancer Biother Radiopharm. 2008;23:43-52.

31. Vidal-Sicart S, Paredes P, Zanon G, et al. Added value of intraoperative real-time imaging in searches for difficult-to-locate sentinel nodes. J Nucl Med. 2010; 51:1219-1225.

32. Vermeeren L, Valdes Olmos RA, Klop WM, Balm AJ, van den Brekel MW. A portable gamma-camera for intraoperative detection of sentinel nodes in the head and neck region. J Nucl Med. 2010;51:700-703.

33. Vermeeren L, Meinhardt W, van der Poel HG, Valdes Olmos RA. Lymphatic drainage from the treated versus untreated prostate: feasibility of sentinel node biopsy in recurrent cancer. Eur J Nucl Med Mol Imaging. 2010;37:2021-2026.
34. Vermeeren L, Valdes Olmos RA, Meinhardt W, et al. Intraoperative radioguidance with a portable gamma-camera: a novel technique for laparoscopic sentinel node localisation in urological malignancies. Eur J Nucl Med Mol Imaging. 2009;36:1029-1036.

35. Machin D, Campbell MJ, Frayers PM, Pinol APY. Sample Size Tables for Clinical Trials. 2nd ed. Cambridge, MA: Blackwell Science; 1997.

36. Lu Y, Bean JA. On the sample size for one-sided equivalence of sensitivities based upon McNemar's test. Stat Med. 1995;14:1831-1839.

37. Fernández MM, Benlloch JM, Cerdá J, et al. A flat-panel-based mini gammacamera for lymph nodes studies. Nucl Instrum Methods Phys Res A. 2004;527: 92-96.

38. Menard L, Charon Y, Solal M, et al. POCI: a compact high resolution gammacamera for intra-operative surgical use. IEEE Trans Nucl Sci. 1998;45:12931297.

39. Aarsvold JN, Greene CM, Mintzer RA, et al. Intraoperative gamma imaging of axillary sentinel lymph nodes in breast cancer patients. Phys Med. 2006;21 (suppl 1):76-79.

40. Goto M, Okuyama C, Kubota T, Ushijima Y, Nishimura T. Assessment of the solid-state gamma-camera to depict axillary sentinel lymph nodes in breast cancer patients. Ann Nucl Med. 2005;19:627-631. 